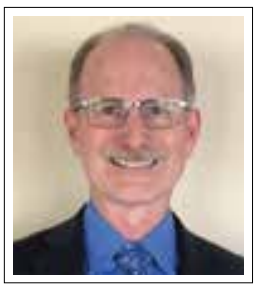

John Hickner, MD, MSc Editor-in-Chief

doi: $10.12788 / \mathrm{jfp} .0310$

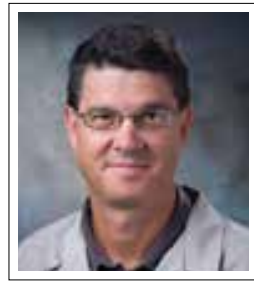

Richard Guthmann, MD, MPH

Advocate Illinois Masonic Family Medicine Residency, Chicago

The authors reported no potential conflict of interest relevant to this editorial. Dr. Guthmann served as the Deputy Editor for the Family Physicians Inquiries Network (FPIN) in the preparation of the Clinical Inquiry discussed here.

\title{
When the evidence suggests that placebo is best
}

I $\mathrm{n}$ this issue of JFP, the Clinical Inquiry on page 461 seeks to answer the question: What are effective injection treatments for lateral epicondylitis? Answering this question proved to be a daunting task for the authors. The difficulty lies in answering this question: effective compared to what?

The injections evaluated in their comprehensive review-corticosteroids, botulinum toxin, hyaluronic acid, platelet-rich plasma, prolotherapy, and autologous blood-have been compared in randomized trials to each other, usual treatment, no treatment, nonmedication treatments, noninjection treatments, surgeries, braces,

\section{What they discovered is that no type of injection therapy has been proven to be better than a saline injection.} and physical therapy. ${ }^{1}$ But which comparison is the best one to determine true effectiveness beyond a placebo effect?

I There are $\mathbf{2}$ choices for an ideal comparison group. One choice compares the active intervention to an adequate placebo, the other compares it to another treatment that has previously been proven effective. Ideally, the other treatment would be a "gold standard" - that is, the best treatment currently available. Unfortunately, for treatment of lateral epicondylitis, no gold standard has been established.

So, what is an "adequate placebo" for injection therapy? This is a very difficult question. The placebo should probably include putting a needle into the treatment site and injecting a nonactive substance, such as saline solution. This is the comparison group Vukelic et al chose for their review. But even saline could theoretically be therapeutic.

Another fair comparison for the treatment of lateral epicondylitis would be an injection near, but not at, the lateral epicondyle. Yet another comparison-dry needling without any medication to the lateral epicondyle vs dry needling of an adjacent location-would also be a fair comparison to help understand the effect of needling alone. Unfortunately, these comparisons have not been explored in randomized controlled trials. Although several studies have evaluated dry needling for lateral epicondylitis, ${ }^{2-4}$ none have used a fair comparison.

Some studies ${ }^{1}$ evaluating treatments for lateral epicondylitis used comparisons to agents that are ineffective or of uncertain effectiveness. Comparing 1 agent to another ineffective or potentially harmful agent obscures our knowledge. Evidencebased medicine must be built on a reliable foundation.

Vukelic and colleagues did an admirable job of selecting studies with an appropriate comparison group - that is, saline injection, the best comparator that has 
CONTINUED FROM PAGE 419

been studied. What they discovered is that no type of injection therapy has been proven to be better than a saline injection.

So, if your patient is not satisfied with conservative therapy for epicondylitis and wants an injection, salt water seems as good as anything.

JFP

1. Sims S, Miller K, Elfar J, et al. Non-surgical treatment of lateral epicondylitis: a systematic review of randomized controlled trials. Hand (NY). 2014;9:419-446. doi: 10.1007/s11552-014-9642-x

2. Uygur E, Aktas B, Ozkut A, et al. Dry needling in lateral epicondylitis: a prospective controlled study. Int Orthop. 2017; 41: 2321-2325. doi: 10.1007/s00264-017-3604-1

3. Krey D, Borchers J, McCamey K. Tendon needling for treatment of tendinopathy: A systematic review. Phys Sportsmed. 2015;43: 80-86. doi: 10.1080/00913847.2015.1004296

4. Jayaseelan DJ, Faller BT, Avery MH. The utilization and effects of filiform dry needling in the management of tendinopathy: a systematic review. Physiother Theory Pract. Published online April 27, 2021. doi: 10.1080/09593985.2021.1920076 